\title{
Determinación de condiciones para encapsulación de proteasa mediante electroatomización
}

\author{
Determination of conditions for protease encapsulation by electrospray \\ Yessica Lorena Díaz $M^{1}$.; Johanna A Serna J²; Laura Sofía Torres $V^{3}$.
}

\footnotetext{
1 Ingeniera Agroindustrial. Universidad, La Gran Colombia, Armenia, Colombia, diazmaryessica@miugca.edu.co.

2 Magister en Diseño y Gestión de Procesos, Universidad La Gran Colombia, Armenia, Colombia, sernajimjohanna@miugca. edu.co.

3. Magister en Ingeniería de Alimentos. Universidad La Gran Colombia, Armenia, Colombia, torresvallaura@miugca.edu.co.
}

Citar: DIAZ, Y.; SERNA, J.; TORRES, L. 2016. Determinación de condiciones para encapsulación de proteasa mediante electroatomización. Rev. Cienc. Agr. 33(1):3 - 8.

Fecha recepción: Marzo 15 de $2016 . \quad$ Fecha de aceptación: Mayo 05 de 2016.

\begin{abstract}
RESUMEN
La encapsulación es un método mediante el cual, sustancias bioactivas son introducidas en una matriz para evitar su pérdida y asimismo, facilitar su incorporación en diferentes productos; no obstante, durante este proceso, es preciso tener en cuenta la técnica y las condiciones de proceso para conseguir la formación de capsulas. El objetivo fue determinar las condiciones adecuadas para encapsulación de proteasa mediante electroatomización. La evaluación del proceso se realizó en función de las variables independientes: voltaje, concentración de material de recubrimiento y el flujo de alimentación; además de las variables dependientes: características del espectro y morfología. A las partículas obtenidas se les midió las características morfológicas (microscopía) y de espectro (Raman). Los resultados mostraron que, las condiciones que permitieron la encapsulación fueron las realizadas a un voltaje de $13 \mathrm{kV}$, empleando un material de recubrimiento con un aporte de solidos solubles totales de $55 \%$ y un flujo de alimentación de $0,1 \mathrm{~mL} / \mathrm{h}$; presentando formación de cápsulas esféricas con espectros (Raman) en el intervalo de 200 a $700 \mathrm{~cm}^{-1}$; observando el pico más alto correspondiente a la enzima a $515 \mathrm{~cm}^{-1} \mathrm{y}$ tamaños entre 0,035 y 1,185 $\mu \mathrm{m}$. A partir de los resultados, se infiere que la electroatomización puede ser considerada como una técnica viable para la encapsulación de enzima proteasa, siempre y cuando se establezcan las condiciones de proceso que son propias de cada solución, permitiendo de esta manera el desarrollo de productos (aditivos) que posteriormente puedan llegar a ser incorporados a otros.
\end{abstract}

Palabras clave: compuestos bioactivos, electrohilado, inmovilización de enzimas, microencapsulación. 


\begin{abstract}
Encapsulation is a method by which bioactive substances are introduced into a matrix to prevent their loss and also facilitate their incorporation into various products; notwithstanding, during this process, it is necessary to take into account technical and process conditions so as to achieve the formation of capsules. The objective of this research was to determine the appropriate conditions for protease encapsulation by electrospray. The process was evaluated according to the independent variables: voltage, concentration of coating material and feeding flow, in addition to dependent variables: spectral characteristics and morphology. In the obtained particles, morphological characteristics (microscopy) and specter (Raman) were measured. The results showed that the conditions allowing the encapsulation by electrospray were those performed at a voltage of $13 \mathrm{~kW}$, using a coating material with a contribution of total soluble solids $55 \%$, and a feeding flow of $0.1 \mathrm{~mL} / \mathrm{h}$, showing formation of spherical capsules with specters (Raman) in the range of 200 to $700 \mathrm{~cm}^{-1}$, observing the highest peak, corresponding to the enzyme at $515 \mathrm{~cm}^{-1}$ and sizes between 0.035 and $1.185 \mu \mathrm{m}$. From the results, is concluded that the electrospray may be considered as a viable technique for encapsulation of protease enzyme, provided that process conditions specific to each solution are established, thereby enabling the development of products (additives) which may be subsequently incorporated into other ones.
\end{abstract}

Key words: Bioactive compounds, electrospinning, immobilization of enzymes, microencapsulation.

\section{INTRODUCCIÓN}

Los procesos de transformación que se aplican a alimentos de consumo humano o animal pueden modificar su composición nutricional, por lo que se requiere desarrollar y/o implementar técnicas que mitiguen esta problemática y permitan ofrecer productos de calidad y con alto valor agregado. En este contexto, una forma de hacerlo, podría ser incorporando nuevamente dichos componentes en las matrices alimentarias ya procesadas; sin embargo, tales componentes requieren de una adecuación que involucra métodos que promueven el mejoramiento tanto de su estabilidad como de su biodisponibilidad (Olagnero et al., 2007; Olagnero et al., 2007; Cruzado y Cedrón, 2012).

Dentro de estos métodos se encuentra la encapsulación, que es un proceso mediante el cual una sustancia (agente activo) es introducida en un material de soporte, una matriz o sistema pared constituida por compuestos poliméricos con el objetivo de impedir su pérdida, protegiéndola del ambiente, de la reacción con otros constituyentes alimentarios o para impedir que sufra reacciones de oxidación debido a la luz o al oxígeno, por lo anterior, la encapsulación es una herramienta útil para mejorar la entrega de alimentos con moléculas bioactivas tales como antioxidantes, minerales, vitaminas, fitoesteroles, luteína, ácidos grasos y el licopeno, al igual que con células vivas como los probióticos y enzimas; de esta manera, se obtienen cápsulas que liberan su contenido a velocidades controladas durante períodos prolongados y en condiciones específicas (Gil y Ruiz, 2010; Nedovic et al., 2011).

Existen diferentes técnicas para encapsular, entre ellas la electroatomización que no emplea altas temperaturas que deterioren componentes termosensibles en el material de interés, con esta técnica, se pueden obtener fibras a escala micro y nanométrica, por medio de estiramiento coaxial de una solución viscoelástica que al ser sometida a fuerzas eléctricas que superen las fuerzas de la tensión superficial en la solución, forman de este modo a un voltaje determinado, finos chorros de solución que son expulsados desde el capilar hasta el plato colector (Duque et al., 2013). Así pues, en los últimos años las investigaciones han ido direccionadas a estudios que determinen la ejecución adecuada y la aplicabilidad que tiene en los ámbi- 
tos multidisciplinares, resaltando la encapsulación de matrices como ingredientes alimentarios (Fathi et al., 2014), polifenoles (Gómez et al., 2015), cúrcuma (Gómez et al., 2015), ácido fólico (Pérez et al., 2015), proteínas (Zamani et al., 2014), polímeros con moléculas terapéuticas (Bock et al., 2012), ciclodextrinas (Saallah et al., 2014), entre otros.

Acorde con lo anterior, el objetivo de la investigación fue determinar las condiciones adecuadas para la encapsulación de proteasa mediante electroatomización.

\section{MATERIALES Y MÉTODOS}

La presente investigación fue realizada en el laboratorio de óptica de la École de Technologie Supérieure de Montreal en la provincia de Quebec en Canadá $\left(23^{\circ} \mathrm{C}, 60 \%\right.$, clima, $31 \mathrm{msnm}$, 4530`29”LN, 73³3`18”LO).

Encapsulación mediante electroatomización. Para la encapsulación por electroatomización se siguió el esquema de proceso reportado por Zamani et al. (2014). En el proceso, se empleó como material de recubrimiento soluciones de maltodextrina grado alimentario con dextrosa equivalente (DE) de 17 (TECNAS, Colombia) con diferentes aportes de solidos solubles totales, como agente a encapsular se utilizó una solución al 10\% (p/v) de la enzima papaína MT80 (Proenzimas, Colombia). Las soluciones se colocaron en 2 jeringas de $5 \mathrm{~mL}$ con aguja de acero inoxidable (Nordson, E.U.A). A la jeringa que contenía la enzima se le conectó un alambre de politetrafluoroetileno que se comunicaba con la jeringa que contenía la matodextrina. Las jeringas se ubicaron en una bomba de jeringa de control digital (KD Scientific, USA) en las que se ajustaron diferentes flujos. La aguja se colocó verticalmente hacia un plato de cobre utilizado como colector. El equipo de electroatomización fue equipado con una fuente de alimentación de alta tensión (Stanford Research System, USA). En la electroatomización la variable de respuesta es la formación del cono de Taylor que se da en función de las variables independientes del proceso: voltaje, concentración de material de recubrimiento y el flujo de alimentación, las cuales se variaron durante el proceso (Tabla 1) con el fin de determinar a qué condiciones se obtuvieron microcápsulas y a las que se les evaluó características del espectro y morfología.

Tabla 1. Parámetros de encapsulación mediante electroatomización.

\begin{tabular}{c|c|c}
\hline $\begin{array}{c}\text { Material de } \\
\text { recubrimiento } \\
\text { SST (\%) }\end{array}$ & $\begin{array}{c}\text { Voltaje } \\
\mathbf{( k V )}\end{array}$ & $\begin{array}{c}\text { Flujo de } \\
\text { alimentación } \\
\text { (mL/h) }\end{array}$ \\
\hline & 9 & \\
& 10 & 0,01 \\
& 11 & 0,02 \\
9 & 12 & 0,03 \\
31 & 13 & 0,04 \\
45 & 14 & 0,05 \\
55 & 15 & 0,06 \\
61 & 16 & 0,07 \\
& 17 & 0,08 \\
& 18 & 0,09 \\
& 19 & 0,1 \\
\hline
\end{tabular}

Las partículas obtenidas fueron evaluadas para determinar si hubo encapsulación de la enzima, adicionalmente se midieron las características del espectro (RAMAN), la morfología y el tamaño de las microcápsulas (SEM).

Espectroscopia de Raman. La caracterización del espectro de la enzima proteasa y de la maltodextrina se hizo a través de espectroscopia de Raman utilizando un equipo Jobin-Yvon IHR320 (Horiba, USA) estabilizado con un TORUS (Laser-Quantum Inc.) con intervalos de longitud de onda entre $200 \mathrm{y}$ $700 \mathrm{~nm}$, potencia nominal de $250 \mathrm{~mW}$ y objetivo de 10 y 40X. La captura de imágenes se realizó con una cámara digital (Nikon Instruments Inc., EE.UU.) de 5 megapíxeles refrigerado con un mecanismo de Peltier adjuntado al microscopio (Mesa et al., 2013). 
Microscopía óptica. Las muestras obtenidas del colector se llevaron a microcopia óptica en un microscopio digital (Nikon eclipse 90i, USA) ajustada a $12 \mathrm{kV}$, con una lámpara halógena de 100W y equipada con un cabezal de imagen digital que integra una iluminación de epifluorescencia (Nikon DS-5mC, USA). La captura de imágenes de realizó con una cámara digital (Nikon Instruments Inc., EE.UU.) de 5 megapíxeles refrigerado con un mecanismo de Peltier adjuntado al microscopio.

Microscopía electrónica de barrido. La morfología de las partículas obtenidas en la encapsulación por electroatomización, se examinaron mediante microscopía electrónica de barrido (SEM) a $5 \mathrm{Kw}$ en un microscopio (HITACHI SU-70, USA). Los diámetros de la cápsula se midieron por medio del software ImageJ 1,45k (versión demo) empleando las micrografías SEM en su magnificación original.

Análisis estadístico. Se trabajó un diseño factorial completamente al azar, los factores evaluados fueron: campo electromagnético y flujo de alimentación. Los resultados referentes a los tamaños de partícula obtenidos y la frecuencia con que se presentaron, fueron sometidos a un Análisis de Varianza (ANDEVA) a un nivel de significancia del 95\%, utilizando el software estadístico Statgraphics ${ }^{\circledR}$ Centurion XVI.II.

\section{RESULTADOS Y DISCUSIÓN}

Encapsulación mediante electroatomización. Las condiciones del proceso que permitieron la formación del cono de Taylor y obtención de microcápsulas fueron las que se ejecutaron empleando un voltaje de $13 \mathrm{kV}$, un material de recubrimiento con un aporte de SST de 55\% y un flujo de alimentación de $0,1 \mathrm{~mL} / \mathrm{h}$, por lo cual son establecidas como condiciones adecuadas para la encapsulación de proteasa a través de electroatomización. Esto confirma lo reportado por Fathi et al. (2014) quien plantea que dichas condiciones deben ser establecidas y controladas, puesto que afectan la formación de cápsulas y su estructura.
Es preciso tener en cuenta que las capsulas formadas, fueron utilizadas en los análisis de espectroscopia de Raman, microscopía óptica y microscopía electrónica de barrido.

Espectroscopia de Raman. Por medio de espectroscopia Raman se identificaron las características del espectro del material de recubrimiento (Shell) y de la enzima proteasa (Core), se encontraron los picos característicos para cada una de las sustancias en el intervalo de 200 y $700 \mathrm{~cm}^{-1}$, se evidenció que el pico más representativo correspondiente a la proteasa se encuentra a $515 \mathrm{~cm}^{-1}$ (Figura 1.)

Microscopía óptica. En las micrografías de microscopía óptica realizadas a las partículas obtenidas, se observa el contraste de los espectros identificados por RAMAN tanto de la enzima encapsulada como de la maltodextrina que la recubre, indicando que la matriz de recubrimiento condujo efectivamente a la formación de cápsulas esféricas dentro de las cuales se observa la enzima, lo que confirma que se produjo encapsulación de la misma. Estos resultados confirman lo reportado en la literatura en donde se expone que la electroatomización permite encapsular o atrapar sustancias en materiales matrices (Duque et al., 2013; Saallah et al., 2014; Gamboa et al., 2007).

Microscopía electrónica de barrido (SEM). Las micrografías SEM correspondientes a las capsulas obtenidas a través de electroatomización se muestran en la Figura 2, en ellas se observa la formación de cápsulas en escala micro cuyo

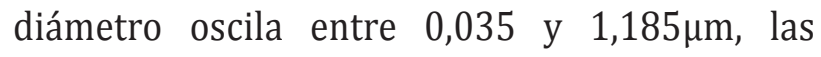
cuales se encuentran dentro del rango de $50 \mathrm{~nm}$ a $10 \mu \mathrm{m}$ establecido para dicha técnica reportado por Gamboa et al. (2017) y de igual forma son similares a las obtenidas mediante la misma técnica utilizando otras matrices, como en el caso de las cápsulas conseguidas encapsulando polifenoles (Gómez et al., 2015) y cúrcuma (Gómez et al., 2015) que fueron de 0,07 a 2,00 $\mu \mathrm{m}$ y $150 \mathrm{~nm}$ a $1,2 \mu \mathrm{m}$, respectivamente. 


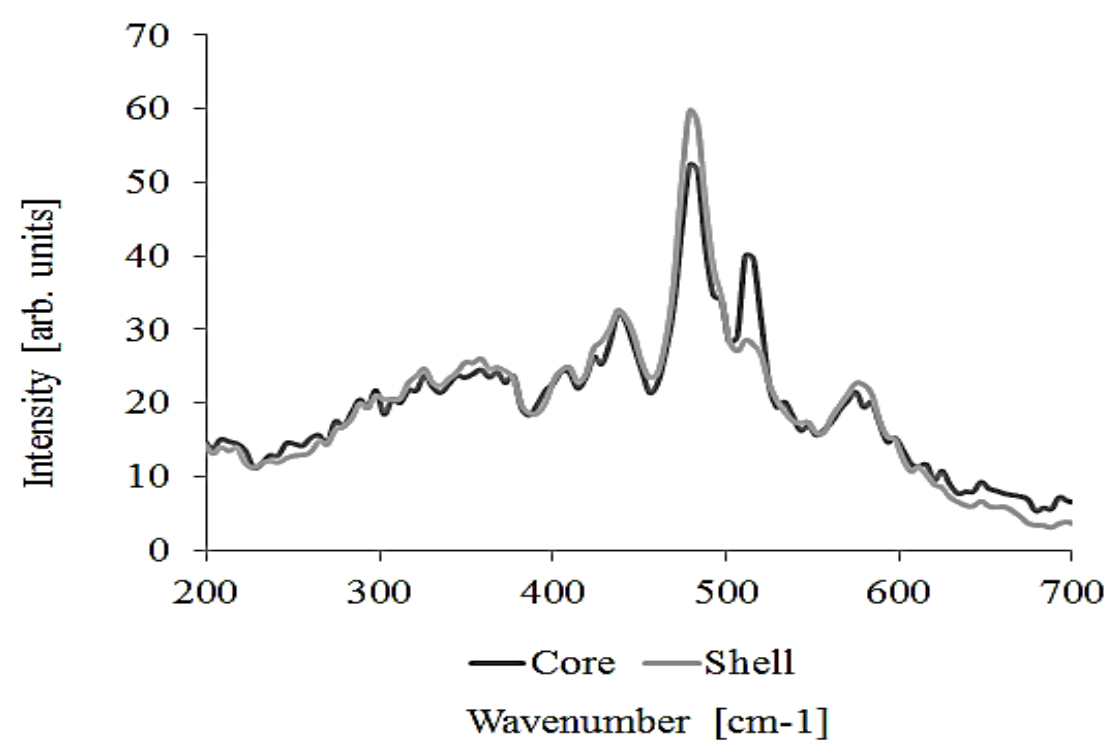

Figura 1. Características del espectro de la enzima y la maltodextrina.

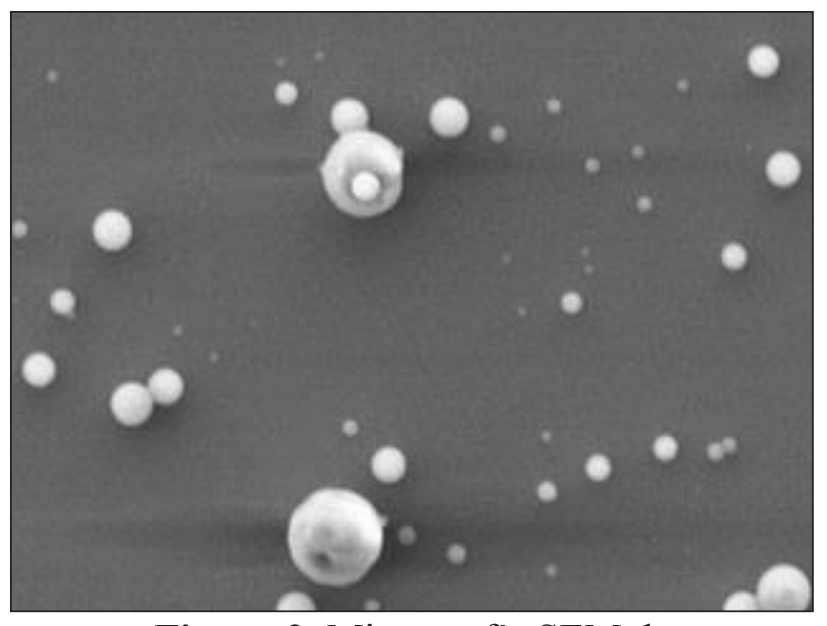

Figura 2. Micrografía SEM de enzima encapsulada.

No obstante, si se contrastan con el diámetro y la morfología de las cápsulas obtenidas mediante otras técnicas de encapsulación como el secado por atomización que son en su mayoría ovaladas y con tamaños de 20 a $200 \mu$ m (López, 2010), se puede evidenciar que las obtenidas mediante electroatomización, son de menor tamaño y de forma esférica, característica que según Pérez et al. (2015) podría favorecer la incorporación y dispersión de las cápsulas dentro de los productos alimenticios sin afectar sus características de textura.
Los resultados de este estudio difieren de los hallados en otras investigaciones y esto se debe a las variables utilizadas en el proceso que intervienen de manera directa en la formación de partículas tales como, la técnica, la temperatura, el campo eléctrico (voltaje y distancia entre cargas), la distancia de inyección, las características de la solución, la velocidad del flujo, la concentración y tipo de material de recubrimiento empleados (Gamboa et al., 2007).

Adicional a esto, se encontró que no hubo una variabilidad estadísticamente significativa en los tamaños de partículas obtenidos en el proceso de electroatomización, llevado a cabo a un voltaje de $13 \mathrm{kV}$, con un material de recubrimiento que aportaba 55\% de SST y un flujo de alimentación de $0,1 \mathrm{~mL} / \mathrm{h}$, a un nivel de confianza del $95 \%$, indicando con esto que las capsulas son homogéneas.

\section{CONCLUSIONES}

La electroatomización es una técnica que permite la encapsulación en enzimas y generan características morfológicas adecuadas para garantizar la estabilidad de los componentes y la posible inclusión en matrices alimentarias que permitan 
la funcionalización de los alimentos, así mismo pueden incluirse en matrices de tipo farmacéutica, nutracéutica y/o agropecuaria.

\section{AGRADECIMIENTOS}

Los autores expresan sus agradecimientos al departamento administrativo de ciencias, tecnología e información COLCIENCIAS, por la financiación del proyecto y al laboratorio de óptica de la Échole de Technologie Supérieure de Montreal, Canadá.

\section{REFERENCIAS BIBLIOGRÁFICAS}

BOCK, N.; DARGAVILLE, T.R.; WOODRUFF, M.A. 2012. Electrospraying of polymers with therapeutic molecules: State of the art. Prog. Polym. Sci. . 37(11):1510 1551. doi:10.1016/j.progpolymsci.2012.03.002.

CRUZADO, M.; CEDRÓN. J.C. 2012. Nutracéuticos, alimentos funcionales y su producción. Rev. Quím. PUCP. 26(1-2):33 - 36.

DUQUE-, L.M.; RODRIGUEZ, L.; LÓPEZ, M. 2013. Electrospinning: La era de las nanofibras. Rev. Iberoam. Polim. 14(1):10 - 27.

FATHI, M.; MARTÍN, Á.; McCLEMENTS, D.J. 2014. Nanoencapsulation of food ingredients using carbohydrate based delivery systems. Trends Food Sci. Technol. 39(1): 18 - 39. doi:10.1016/j.tifs.2014.06.007

GAMBOA, W.; MANTILLA, 0.; CASTILLO, V. 2007. Producción de micro y nano fibras a partir de la técnica "Electrospinning" para aplicaciónes farmacológicas. pp. 4. En: Memorias VII Congreso Sociedad Cubana de Bioingeniería. Habana, Cuba.

GÓMEZ, J.; GAVARA, R.; HERNÁNDEZ, P. 2015. Encapsulation of curcumin in electrosprayed gelatin microspheres enhances its bioaccessibility and widens its uses in food applications. Innov. Food Sci. Emerg. Technol. 29:301 - 307. doi:10.1016/j.ifset.2015.03.004

GÓMEZ-, L.G.; LAGARÓN, J.M.; LÓPEZ, A. 2015. Electrosprayed gelatin submicroparticles as edible carriers for the encapsulation of polyphenols of interest in functional foods. Food Hydrocoll. 49: 42 - 52. doi:10.1016/j. foodhyd.2015.03.006
GIL, A.; RUIZ, M.D. 2010. Tratado de Nutrición: Composición y Calidad Nutritiva de los Alimentos. Segunda edición. Editorial Medica Panamericana. Madrid, España, 812 p.

LÓPEZ, O.D. 2010. Microencapsulación de sustancias oleosas mediante secado por aspersión. Rev. Cubana Farm. 44(3):381 - 389.

MESA, F.A.; CUÉLLAS, A.; PERILLA, J.E.; VARGAS, C. 2013. Caracterización de los monómeros DGEBA y TMAB, y seguimiento de la reacción de entrecruzamiento por espectroscopia Raman. Ingeniare. Rev. Chil. Ing. 21(3):414 - 423.

NEDOVIC, V.; KALUSEVIC, A.; MANOJLOVIC, V.; LEVIC, S.; BUGARSKI, B. 2011. An overview of encapsulation technologies for food applications. Procedia Food Sci. 1:806 - 1815. doi:10.1016/j.profoo.2011.09.265

OLAGNERO, G.; ABAD, A.; BENDERSKY, S.; GENEVOIS, C.; GRANZELLA, L.; MONTONATI, M. 2007. Alimentos funcionales: fibra, prebióticos, probióticos y simbióticos. DIAETA. 25(121):20 - 33

OLAGNERO, G.; GENEVOIS, C.; IREI, V.; MARCENAD, J.; BENDERSKY, S. 2007. Alimentos funcionales: Conceptos, Definiciones y Marco Legal Global. DIAETA. 25(119):33 $-41$

PÉREZ, R.; LÓPEZ, R.; PERIAGO, M.J.; ROS, G.; LAGARÓN, J, M.; LÓPEZ, A. 2015. Encapsulation of folic acid in food hydrocolloids through nanospray drying and electrospraying for nutraceutical applications. Food Chem. 168:124 - 133. doi: http://dx.doi.org/10.1016/j. foodchem.2014.07.051

SAALLAH, S.; NAIM, M.N.; MOKHTAR, M.N.; ABU-BAKAR, N.F.; GEN, M.; LENGGORO, I.W. 2014. Transformation of cyclodextrin glucanotransferase (CGTase) from aqueous suspension to fine solid particles via electrospraying. Enzyme Microb. Technol. 64 - 65: 52 -59. doi:10.1016/j. enzmictec.2014.06.002

ZAMANI, M.; PRABHAKARAN, M.P.; THIAN, E.S.; RAMAKRISHNA, S. 2014. Protein encapsulated core-shell structured particles prepared by coaxial electrospraying: Investigation on material and processing variables. Int. J. Pharm. 473(1-2):134 - 143. doi:10.1016/j.ijpharm.2014.07.006. 